\title{
Association between milk protein gene variants and protein composition traits in dairy cattle
}

\author{
W. Huang, ${ }^{*}$ F. Peñagaricano,† K. R. Ahmad, ${ }^{*}$ J. A. Lucey,‡ K. A. Weigel, ${ }^{*}$ and H. Khatib ${ }^{1}$ \\ *Department of Dairy Science, \\ tDepartment of Animal Sciences, and \\ ¥Department of Food Science, University of Wisconsin-Madison, Madison 53706
}

\begin{abstract}
The objective of this study was to identify DNA markers in the 4 casein genes (CSN1S1, CSN1S2, CSN2, and CSN3) and the 2 major whey protein genes ( $L A L B A$ and $L G B$ ) that show associations with milk protein profile measured by reverse-phase HPLC. Fifty-three single nucleotide polymorphisms (SNP) were genotyped for cows in a unique resource population consisting of purebred Holstein and (Holstein $x$ Jersey) $\times$ Holstein crossbred animals. Seven traits were analyzed, including concentrations of $\alpha_{S^{-}}$casein $(\mathrm{CN})$, $\beta$-CN, $\kappa$-CN, $\alpha$-lactalbumin, $\beta$-lactoglobulin, and 2 additional secondary traits, the total concentration of the above 5 milk proteins and the $\alpha_{S^{-}} \mathrm{CN}$ to $\beta$-CN ratio. A substantial fraction of phenotypic variation could be explained by the additive genetic component for the 7 milk protein composition traits studied. Moreover, several SNP were significantly associated with all examined traits at an experiment-wise error rate of 0.05 , except for $\alpha$-lactalbumin. Importantly, the significant SNP explained a large proportion of the phenotypic variation of milk protein composition. Our findings could be used for selecting animals that produce milk with desired composition or desired processing and manufacturing properties.
\end{abstract}

Key words: milk protein gene, milk protein composition, association, single nucleotide polymorphism

\section{INTRODUCTION}

Milk protein composition influences many aspects of the dairy industry. The protein content of milk, which depends on the expression and secretion of individual proteins, is one of the major determinants of milk price (Bailey et al., 2005). Moreover, protein profile, particularly casein content, has long been known to affect cheese yield (Lucey and Kelly, 1994), and the ratio

Received July 22, 2011.

Accepted September 5, 2011.

${ }^{1}$ Corresponding author: hkhatib@wisc.edu of $\alpha_{S^{-}} \mathrm{CN}$ to $\beta-\mathrm{CN}$ is important for rennet coagulation (St-Gelais and Hache, 2005), suggesting that not only do individual protein contents influence cheese making, but the relative amounts among them also matter. Furthermore, protein composition affects the nutritional profile of milk by altering its amino acid composition and the availability of bioactive peptides. Indeed, a variety of bioactive peptides present in caseins and whey proteins are known to have important human health implications (e.g., Tauzin et al., 2002).

Many factors affect milk protein composition, including breed (Auldist et al., 2004), season, and lactation stage (Auldist et al., 1998), as well as genetic polymorphisms. Indeed, a significant genetic component exists for milk protein concentrations. The intraherd heritabilities for individual protein fractions of total milk protein, as estimated by Schopen et al. (2009), were moderate to high ( 0.25 for $\beta-\mathrm{CN}$ to 0.80 for $\beta-\mathrm{LG}$ ). In particular, polymorphisms within the major milk protein genes have been found to be associated with protein composition, a finding that has been replicated in different populations and studies. For example, the A variant of $\beta-L G$ has been repeatedly found to be associated with higher $\beta$-LG concentration (Ng-Kwai-Hang et al., 1987; Heck et al., 2009).

However, most previous studies on genetic associations between milk protein polymorphisms and individual protein fractions have investigated a limited number of polymorphic sites. Historically, these genetic variants were identified by protein analysis. Thus, they represent non-synonymous variations in the coding sequences of the milk protein genes (Caroli et al., 2009). Nevertheless, milk protein genes are highly polymorphic, containing an unusually large number of polymorphisms (e.g., Nilsen et al., 2009). In this study, a comprehensive association analysis between DNA variants of milk protein genes and milk protein profile measured by reverse-phase HPLC is reported in a resource population consisting of both purebred Holstein and (Holstein $\times$ Jersey) $\times$ Holstein backcross cows. Because a substantial difference exists in milk protein content between Holstein and Jersey, the introduction 
of Jersey alleles to the mapping population is expected to generate genetic variation, which in turn facilitates the mapping of DNA variants responsible for milk protein composition traits.

\section{MATERIALS AND METHODS}

\section{Milk Sampling}

Milk samples were collected from lactating cows (60 to $240 \mathrm{~d}$ in milk in the first 3 lactations) at the University of Wisconsin-Madison Arlington Agriculture Experimental Station. All animals were housed under the same conditions and milked twice daily. Milk samples were centrifuged at $1,000 \times g$ for $15 \mathrm{~min}$ to remove fat, aliquoted, and stored at $-80^{\circ} \mathrm{C}$ before they were analyzed. Of the 283 cows that were sampled, 103 were purebred Holsteins and 180 were crossbreds generated by backcrossing $F_{1}$ Holstein $\times$ Jersey sires to Holstein dams as described previously (Maltecca et al., 2009).

\section{Phenotyping}

The concentrations of 5 major milk proteins $\left(\alpha_{S^{-}} \mathrm{CN}\right.$, $\beta-\mathrm{CN}, \kappa-\mathrm{CN}, \alpha-\mathrm{LA}, \beta-\mathrm{LG})$ were measured by reverse phase HPLC using a previously described procedure with some modifications (Wedholm et al., 2006). Because $\alpha_{S_{2}}-\mathrm{CN}$ cannot be readily distinguished from $\alpha_{\mathrm{S} 1}-\mathrm{CN}$ by HPLC and lacks standard purified protein, a combined $\alpha_{S}-\mathrm{CN}$ concentration was obtained. Briefly, $80 \mu \mathrm{L}$ of defatted milk was incubated with $320 \mu$ Lof freshly made reducing buffer $(20 \mathrm{~m} M$ EDTA and $8 M$ urea) at room temperature for $1.5 \mathrm{~h}$. Reduced and denatured milk was diluted by adding $1.2 \mathrm{~mL}$ of buffer A (water:acetonitrile:trifluoroacetic acid, 90:10:0.1) and filtered through a $0.22-\mu \mathrm{m}$ polyvinylidene fluoridelowprotein binding syringe filter. Each filtered sample (30 $\mu \mathrm{L}$ ) was injected into a $\mathrm{C} 4$ protein column (Vydac, Deerfield, IL) to begin the HPLC run. The mobile phase of the column consisted of a gradient mixture of buffer A and buffer B (water:acetonitrile:trifluoroacet ic acid, 10:90:0.1) at a flow rate of $0.3 \mathrm{~mL} / \mathrm{min}$. The elution began with $33 \%$ buffer B for 12 min, followed by a linear gradient to $35 \% \mathrm{~B}$ in $4 \mathrm{~min}, 35 \% \mathrm{~B}$ for 7 min, $35 \%$ to $37 \%$ B in $7 \mathrm{~min}, 37 \%$ B for $5 \mathrm{~min}, 37 \%$ to $43 \% \mathrm{~B}$ in $15 \mathrm{~min}$, and a final isocratic elution at 43\% B for $10 \mathrm{~min}$. Protein eluate was detected by a PDA996 UV detector (Waters, Milford, MA) at 215 nm wavelength. The HPLC column was maintained at room temperature and all results were analyzed by using the Millennium software (Waters).

To quantify individual proteins, standard purified proteins $\left(\alpha_{S}-\mathrm{CN}, \beta-\mathrm{CN}, \kappa-\mathrm{CN}, \alpha-\mathrm{LA}\right.$, and $\left.\beta-\mathrm{LG}\right)$ were obtained from Sigma (St. Louis, MO). Standard proteins were reduced in $400 \mu \mathrm{L}$ of reducing buffer and then mixed and processed in the same way as milk samples. Standard curves were obtained by running serially diluted standard protein mixtures in duplicate. All standard curves had a correlation $>0.99$. Concentrations of individual milk proteins were determined by integration of the chromatograms and interpolation using the standard curves. For each animal, one replicate of HPLC measurement was performed and 7 traits were measured and calculated, including concentrations (in $\mathrm{mg} / 100 \mu \mathrm{L})$ of $\alpha_{\mathrm{S}}-\mathrm{CN}, \beta-\mathrm{CN}, \kappa-\mathrm{CN}, \alpha-\mathrm{LA}, \beta-\mathrm{LG}$, and 2 additional secondary traits, total concentration of the above 5 major milk proteins (total protein) and $\alpha_{S^{-}}$CN to $\beta-\mathrm{CN}$ ratio. Summary statistics for the 7 traits are presented in Table 1.

\section{Genotyping}

Genomic DNA was extracted from white blood cells of animals by proteinase $\mathrm{K}$ digestion, phenol/chloroform extraction, and isopropanol precipitation. The DNA was genotyped using an iPlex assay (Sequenom, San Diego, CA) by the service provider GeneSeek (Lincoln, $\mathrm{NE}$ ). For genotyping, $80 \mathrm{SNP}$ were compiled from published sources. The chosen SNP were within and near the milk protein genes including CSN1S1 (encoding the $\alpha_{\left.S_{1}-\text { casein }\right), C S N 1 S 2}\left(\alpha_{S_{2}-} \mathrm{CN}\right), C S N 2(\beta-\mathrm{CN}), C S N 3$ ( $\kappa-\mathrm{CN}), L A L B A(\alpha-\mathrm{LA})$, and $L G B(\beta-\mathrm{LG})$. Only SNP with minor allele frequencies $>0.05$ and at least $90 \%$ call rate were analyzed further. Fifty-three SNP met

Table 1. Summary statistics of 7 milk protein composition traits ${ }^{1}$

\begin{tabular}{lccc}
\hline Trait & $\begin{array}{c}\text { Holsteins } \\
(\mathrm{n}=103)\end{array}$ & $\begin{array}{c}\text { Crossbreds } \\
(\mathrm{n}=180)\end{array}$ & $\begin{array}{c}\text { All } \\
(\mathrm{n}=283)\end{array}$ \\
\hline$\alpha_{\mathrm{S}} \mathrm{CN}(\mathrm{mg} / 100 \mu \mathrm{L})$ & $0.8334(0.0993)$ & $0.9092(0.1053)$ & $0.8833(0.1092)$ \\
$\beta-\mathrm{CN}(\mathrm{mg} / 100 \mu \mathrm{L})$ & $1.2826(0.1681)$ & $1.3675(0.1525)$ & $1.3386(0.1628)$ \\
$\kappa-C N(\mathrm{mg} / 100 \mu \mathrm{L})$ & $0.4033(0.0690)$ & $0.4341(0.0802)$ & $0.4236(0.0778)$ \\
$\alpha-\mathrm{LA}(\mathrm{mg} / 100 \mu \mathrm{L})$ & $0.1166(0.0165)$ & $0.1188(0.0146)$ & $0.1180(0.0153)$ \\
$\beta-\mathrm{LG}(\mathrm{mg} / 100 \mu \mathrm{L})$ & $0.4673(0.1096)$ & $0.5539(0.1193)$ & $0.5244(0.1230)$ \\
Total protein $(\mathrm{mg} / 100 \mu \mathrm{L})$ & $3.1032(0.3589)$ & $3.3835(0.3746)$ & $3.2879(0.3920)$ \\
$\alpha_{S^{-}} \mathrm{CN}$ to $\beta-\mathrm{CN}$ ratio & $0.6521(0.0418)$ & $0.6658(0.0424)$ & $0.6611(0.0426)$ \\
\hline
\end{tabular}

${ }^{1}$ Data are expressed as mean (SD). 
these criteria and a full description including alleles, minor allele frequency, physical map location, location relative to genes, AA change associated with protein variants, dbSNP (http://www.ncbi.nlm.nih.gov/projects/SNP/) ID in the NCBI SNP database if available, and references are provided in Table 2 . Linkage disequilibrium (LD) between SNP in the mapping population is shown in Figure 1. The LD heatmap (Figure 1) was created using Haploview (Barrett et al., 2005).

\section{Statistical Analysis}

Each of the 7 traits (concentrations of $\alpha_{S^{-}} \mathrm{CN}, \beta-\mathrm{CN}$, $\kappa-\mathrm{CN}, \alpha-\mathrm{LA}, \beta-\mathrm{LG}$, total protein, and $\alpha_{\mathrm{S}}-\mathrm{CN}$ to $\beta-\mathrm{CN}$ ratio) was modeled using a mixed effects model:

$$
\begin{gathered}
\mathrm{y}_{\mathrm{ijklmn}}=\mathrm{b}_{0}+\text { Breed }_{\mathrm{i}}+\mathrm{b}_{1} \text { Lactation }_{\mathrm{j}}+\mathrm{b}_{2} \mathrm{DIM}_{\mathrm{k}} \\
+\mathrm{b}_{3}\left(\mathrm{DIM}_{\mathrm{k}}\right)^{2}+\mathrm{SNP}_{1}+\text { Date }_{\mathrm{m}}+\text { Animal }_{\mathrm{n}}+\mathrm{e}_{\mathrm{ijlkmn}},
\end{gathered}
$$

where $y_{i j k l m n}$ is the trait being modeled; the constant $\mathrm{b}_{0}$ represents a population mean; Breed $\mathrm{i}_{\mathrm{i}}$ is the fixed effect of breed i (Holstein or crossbreed); $b_{1}$ is the effect of lactation number; $b_{2}$ and $b_{3}$ are the linear and quadratic coefficients of DIM, respectively; $\mathrm{SNP}_{1}$ is the fixed effect of the SNP genotype, each SNP was analyzed separately; Date ${ }_{m}$ is the random effect the date of sample collection and analysis, which accounted for seasonal and batch effect on HPLC; Animal $_{n}$ is the additive genetic effect whose covariance structure is specified by a relationship matrix calculated based on the pedigree of animals; and $\mathrm{e}_{\mathrm{ijk} \text { klmn }}$ is a random error with independence and normality assumptions. Pedigrees were recorded for animals and relatives within the herd. Depending on the family history, pedigrees of animals could be traced back between 1 to 5 generations and because common sires were used to breed crossbreds, many animals were half sibs. The additive relationship matrix was calculated as twice as the kinship matrix using the "kinship" package in R (R Development Core Team, 2010). The SNP term was removed when estimating the additive genetic variance. When estimating their variance components, SNP were fitted as random effects. Models were fitted using the MIXED procedure (SAS Institute, 2009). When the number of animals within a genotype of a SNP was less than 5, animals within the genotype were excluded from the analysis.

Significance of the SNP term was tested by a type III $F$-test in the MIXED procedure. Because SNP genotypes and traits were correlated, the nominal $P$ value for multiple testing was corrected by dividing it by an effective number of independent tests. An effective number of markers $\left(\mathbf{M}_{\text {eff }}\right)$ was calculated as the minimum number of principal components explaining at least $99 \%$ of the variation in the genotype (coded as $0,1,2)$ matrix following a previously described procedure (Gao et al., 2008). In this study, $\mathrm{M}_{\text {eff }}=16$ and 7 traits were tested. Although traits were genetically correlated, we conservatively considered that $16 \times 7$ tests were made; thus, a nominal $P$-value of 0.00046 corresponded to an experiment-wise error rate of 0.05 by a Bonferroni/Sidak correction. A plot for $P$-values was created using functions in the software package $\mathrm{R}$ (R Development Core Team, 2010). For each trait, a stepwise selection was performed beginning with the most significant SNP to search for the best subset of SNP among all significant SNP. The threshold for including a SNP in the final model was a nominal $P$-value of 0.001 .

\section{RESULTS}

\section{Additive Genetic Components of Milk Protein Composition Traits}

To investigate the proportion of phenotypic variation that can be attributed to the additive genetic component, pedigree-based additive genetic variance of animals for each trait was estimated using a mixed effects animal model. Out of the total variance after correcting for fixed effects, 33.2, 32.8, 66.3, 32.6, 69.1, 39.6 , and $66.7 \%$ could be attributed to additive genetic variance for $\alpha_{S}-\mathrm{CN}, \beta-\mathrm{CN}, \kappa-\mathrm{CN}, \alpha-\mathrm{LA}, \beta-\mathrm{LG}$, total protein, and $\alpha_{S}-\mathrm{CN}$ to $\beta$-CN ratio, respectively (Table 3 ). Although the magnitude of estimates differed, this result was consistent with previous studies (Schopen et al., 2009, 2011) and indicated a substantial additive genetic component for the milk protein composition traits.

\section{Association Between SNP in Milk Protein Genes and Protein Composition Traits}

The relatively high proportion of genetic variance of protein composition traits provided an opportunity for genetic dissections of these traits. Associations were tested between each of the 7 traits and 42 SNP in the casein cluster on BTA6 covering the genic and intergenic regions of the casein genes CSN1S1, CSN2, CSN1S2, and $C S N 3$; 9 SNP on BTA11 within or near the $L G B$ gene that codes for the $\beta$-LG precursor; and 2 SNP on BTA5 within or near the $L A L B A$ gene that encodes the $\alpha$-LA precursor (Table 2, Figure 1).

Significant associations were found for all examined traits at an experiment-wise error rate of 0.05 , except for $\alpha$-LA (Figure 2). The SNP BTA6.88307280 in CSN1S1 was significantly associated with $\alpha_{S^{-}} \mathrm{CN}$ concentration and $\alpha_{S^{-}} \mathrm{CN}$ to $\beta-\mathrm{CN}$ ratio (Figure 2; Supplemental 
Table 2. Information on polymorphic SNP used in the study

\begin{tabular}{|c|c|c|c|c|c|c|c|c|}
\hline ID & Alleles ${ }^{1}$ & $\mathrm{MAF}^{2}$ & Chromosome & $\begin{array}{c}\text { Position } \\
\text { (on btau4.0) }\end{array}$ & Gene & $\begin{array}{l}\text { Gene } \\
\text { position }\end{array}$ & dbSNP ID & Reference \\
\hline BTA5.34384932 & $\mathrm{G} / \mathrm{C}$ & 0.14 & 5 & 34384932 & $\mathrm{NA}^{3}$ & Intergenic & rs133239284 & Kaminski et al., 2005 \\
\hline BTA5.34386828 & $\mathrm{G} / \mathrm{A}$ & 0.25 & 5 & 34386828 & $L A L B A$ & 5' UTR & $\mathrm{NA}^{4}$ & Kaminski et al., 2005 \\
\hline BTA6.88291433 & $\mathrm{A} / \mathrm{G}$ & 0.18 & 6 & 88291433 & NA & Intergenic & rs109817504 & Nilsen et al., 2009 \\
\hline BTA6.88295268 & $\mathrm{C} / \mathrm{T}$ & 0.40 & 6 & 88295268 & $C S N 1 S 1$ & Intronic & rs110899052 & Nilsen et al., 2009 \\
\hline BTA6.88306150 & $\mathrm{G} / \mathrm{C}$ & 0.40 & 6 & 88306150 & CSN1S1 & Intronic & rs110258698 & Nilsen et al., 2009 \\
\hline BTA6.88307280 & $\mathrm{A} / \mathrm{G}$ & 0.16 & 6 & 88307280 & $C S N 1 S 1$ & Glu (B) - Gly $(\mathrm{C})^{5}$ & rs43703010 & Nilsen et al., 2009 \\
\hline BTA6.88330008 & $\mathrm{A} / \mathrm{G}$ & 0.49 & 6 & 88330008 & CSN2 & Intronic & rs110223653 & Nilsen et al., 2009 \\
\hline BTA6.88330265 & $\mathrm{A} / \mathrm{C}$ & 0.34 & 6 & 88330265 & CSN2 & Intronic & rs110672723 & Nilsen et al., 2009 \\
\hline BTA6.88331153 & $\mathrm{G} / \mathrm{T}$ & 0.33 & 6 & 88331153 & CSN2 & Pro $\left(\mathrm{A}^{2}\right)-\operatorname{His}\left(\mathrm{A}^{1}\right)$ & rs43703011 & Nilsen et al., 2009 \\
\hline BTA6.88332840 & $\mathrm{C} / \mathrm{A}$ & 0.49 & 6 & 88332840 & CSN2 & Intronic & rs109526437 & Nilsen et al., 2009 \\
\hline BTA6.88333146 & $\mathrm{C} / \mathrm{T}$ & 0.31 & 6 & 88333146 & CSN2 & Intronic & rs110564693 & Nilsen et al., 2009 \\
\hline BTA6.88337966 & $\mathrm{T} / \mathrm{C}$ & 0.49 & 6 & 88337966 & CSN2 & Intronic & rs109091301 & Nilsen et al., 2009 \\
\hline BTA6.88339983 & $\mathrm{G} / \mathrm{A}$ & 0.18 & 6 & 88339983 & NA & Intergenic & rs110018851 & Nilsen et al., 2009 \\
\hline BTA6.88340058 & $\mathrm{A} / \mathrm{G}$ & 0.20 & 6 & 88340058 & NA & Intergenic & rs110690977 & Nilsen et al., 2009 \\
\hline BTA6.88378199 & $\mathrm{T} / \mathrm{C}$ & 0.34 & 6 & 88378199 & STATH & Intronic & rs110649731 & Nilsen et al., 2009 \\
\hline BTA6.88407308 & $\mathrm{G} / \mathrm{A}$ & 0.48 & 6 & 88407308 & NA & Intergenic & rs109579742 & Nilsen et al., 2009 \\
\hline BTA6.88408705 & G/A & 0.32 & 6 & 88408705 & NA & Intergenic & rs110158897 & Nilsen et al., 2009 \\
\hline BTA6.88413710 & $\mathrm{T} / \mathrm{C}$ & 0.34 & 6 & 88413710 & CSN1S2 & Intronic & rs110808655 & Nilsen et al., 2009 \\
\hline BTA6.88415825 & $\mathrm{A} / \mathrm{G}$ & 0.16 & 6 & 88415825 & CSN1S2 & Intronic & rs109248332 & Nilsen et al., 2009 \\
\hline BTA6.88419757 & $\mathrm{T} / \mathrm{A}$ & 0.16 & 6 & 88419757 & CSN1S2 & Intronic & rs109557417 & Nilsen et al., 2009 \\
\hline BTA6.88422588 & $\mathrm{T} / \mathrm{C}$ & 0.35 & 6 & 88422588 & CSN1S2 & Intronic & rs109185641 & Nilsen et al., 2009 \\
\hline BTA6.88426653 & $\mathrm{A} / \mathrm{G}$ & 0.34 & 6 & 88426653 & CSN1S2 & Intronic & rs109500451 & Nilsen et al., 2009 \\
\hline BTA6.88427361 & $\mathrm{C} / \mathrm{T}$ & 0.37 & 6 & 88427361 & CSN1S2 & $3^{\prime} \mathrm{UTR}$ & rs109261203 & Nilsen et al., 2009 \\
\hline BTA6.88470657 & $\mathrm{T} / \mathrm{C}$ & 0.13 & 6 & 88470657 & NA & Intergenic & rs41588955 & Nilsen et al., 2009 \\
\hline BTA 6.88470917 & $\mathrm{G} / \mathrm{A}$ & 0.13 & 6 & 88470917 & NA & Intergenic & rs41588953 & Nilsen et al., 2009 \\
\hline BTA6.88505736 & $\mathrm{T} / \mathrm{C}$ & 0.08 & 6 & 88505736 & NA & Intergenic & rs43703014 & Nilsen et al., 2009 \\
\hline BTA6.88508849 & $\mathrm{T} / \mathrm{C}$ & 0.17 & 6 & 88508849 & NA & Intergenic & rs41588950 & Nilsen et al., 2009 \\
\hline BTA6.88508981 & $\mathrm{C} / \mathrm{A}$ & 0.15 & 6 & 88508981 & NA & Intergenic & rs41588946 & Nilsen et al., 2009 \\
\hline BTA6.88509069 & $\mathrm{G} / \mathrm{A}$ & 0.15 & 6 & 88509069 & NA & Intergenic & rs41588945 & Nilsen et al., 2009 \\
\hline BTA6.88509123 & $\mathrm{G} / \mathrm{A}$ & 0.15 & 6 & 88509123 & NA & Intergenic & rs41588944 & Nilsen et al., 2009 \\
\hline BTA6.88519756 & $\mathrm{A} / \mathrm{T}$ & 0.45 & 6 & 88519756 & NA & Intergenic & rs110303451 & Nilsen et al., 2009 \\
\hline BTA6.88521021 & $\mathrm{T} / \mathrm{C}$ & 0.09 & 6 & 88521021 & $N A$ & Intergenic & rs111012468 & Nilsen et al., 2009 \\
\hline BTA6.88528997 & $\mathrm{C} / \mathrm{A}$ & 0.47 & 6 & 88528997 & CSN3 & Intronic & rs110079521 & Nilsen et al., 2009 \\
\hline BTA6.88530211 & $\mathrm{C} / \mathrm{T}$ & 0.39 & 6 & 88530211 & CSN3 & Intronic & rs110888103 & Nilsen et al., 2009 \\
\hline BTA6.88531650 & $\mathrm{A} / \mathrm{G}$ & 0.46 & 6 & 88531650 & CSN3 & Intronic & rs110323127 & Nilsen et al., 2009 \\
\hline BTA6.88532296 & $\mathrm{C} / \mathrm{T}$ & 0.39 & 6 & 88532296 & CSN3 & Thr (A) - Ile (B) & rs43703015 & Nilsen et al., 2009 \\
\hline BTA6.88532332 & $\mathrm{A} / \mathrm{C}$ & 0.39 & 6 & 88532332 & CSN3 & Asp (A) - Ala (B) & rs43703016 & Nilsen et al., 2009 \\
\hline BTA6.88532352 & $\mathrm{A} / \mathrm{G}$ & 0.08 & 6 & 88532352 & CSN3 & Ser $(A)-$ Gly $(E)$ & rs43703017 & Nilsen et al., 2009 \\
\hline BTA6.88532401 & $\mathrm{A} / \mathrm{T}$ & 0.39 & 6 & 88532401 & CSN3 & $3^{\prime} \mathrm{UTR}$ & rs109787476 & Nilsen et al., 2009 \\
\hline BTA6.88532921 & $\mathrm{C} / \mathrm{T}$ & 0.39 & 6 & 88532921 & CSN3 & Intronic & rs110975631 & Nilsen et al., 2009 \\
\hline BTA6.88533203 & $\mathrm{T} / \mathrm{G}$ & 0.38 & 6 & 88533203 & CSN3 & Intronic & rs109663346 & Nilsen et al., 2009 \\
\hline BTA6.88533421 & $\mathrm{G} / \mathrm{A}$ & 0.46 & 6 & 88533421 & CSN3 & Intronic & rs109190605 & Nilsen et al., 2009 \\
\hline BTA6.88533623 & $\mathrm{A} / \mathrm{G}$ & 0.40 & 6 & 88533623 & CSN3 & Intronic & rs109311071 & Nilsen et al., 2009 \\
\hline BTA6.88534063 & $\mathrm{C} / \mathrm{G}$ & 0.40 & 6 & 88534063 & CSN3 & Intronic & rs109344408 & Nilsen et al., 2009 \\
\hline BTA11.107166278 & $\mathrm{G} / \mathrm{C}$ & 0.41 & 11 & 107166278 & NA & Intergenic & rs41255679 & Ganai et al., 2009 \\
\hline BTA11.107166291 & $\mathrm{G} / \mathrm{A}$ & 0.21 & 11 & 107166291 & NA & Intergenic & rs 41255680 & Ganai et al., 2009 \\
\hline BTA11.107166346 & $\mathrm{C} / \mathrm{T}$ & 0.40 & 11 & 107166346 & $\mathrm{NA}$ & Intergenic & rs41255681 & Ganai et al., 2009 \\
\hline BTA11.107167657 & $\mathrm{C} / \mathrm{T}$ & 0.05 & 11 & 107167657 & $L G B$ & Intronic & rs109498821 & Ganai et al., 2009 \\
\hline BTA11.107168522 & $\mathrm{T} / \mathrm{C}$ & 0.45 & 11 & 107168522 & $L G B$ & Synonymous & rs110180463 & Ganai et al., 2009 \\
\hline BTA11.107168524 & $\mathrm{A} / \mathrm{G}$ & 0.41 & 11 & 107168524 & $L G B$ & $\operatorname{Asp}(\mathrm{A})-\operatorname{Gly}(\mathrm{B})$ & rs110066229 & Ganai et al., 2009 \\
\hline BTA11.107169806 & $\mathrm{T} / \mathrm{C}$ & 0.41 & 11 & 107169806 & $L G B$ & $\operatorname{Val}(\mathrm{A})-\mathrm{Ala}(\mathrm{B})$ & rs109625649 & Ganai et al., 2009 \\
\hline BTA11.107170500 & $\mathrm{G} / \mathrm{A}$ & 0.10 & 11 & 107170500 & $L G B$ & Synonymous & rs109029284 & Ganai et al., 2009 \\
\hline BTA11.107171098 & $\mathrm{G} / \mathrm{A}$ & 0.21 & 11 & 107171098 & $L G B$ & Intronic & rs110618886 & Ganai et al., 2009 \\
\hline
\end{tabular}

${ }^{1}$ Alleles are ordered as major allele/minor allele.

${ }^{2}$ Minor allele frequency.

${ }^{3} \mathrm{NA}=\mathrm{SNP}$ not located in gene.

${ }^{4} \mathrm{NA}=$ no dbSNP ID.

${ }^{5}$ Nonsynonymous SNP are indicated by the AA coded by the 2 alleles, and the conventional protein variants identified by the DNA alleles are indicated in parentheses.

Table 1, http://www.journalofdairyscience.org/). Interestingly, the frequency of the $\alpha_{S^{-}} \mathrm{CN}$-increasing $\mathrm{G}$ allele for this SNP differed significantly (Fisher's exact test: $P=1 \times 10^{-7}$ ) between purebred Holstein $(\mathrm{G}$ allele frequency $=0.045, \mathrm{n}=100)$ and Jersey $\times$ Holstein crossbred animals $(\mathrm{G}$ allele frequency $=0.202, \mathrm{n}=$ 186). This was likely the reason that no homozygous $G$ animals were observed because dams of these animals 
(A)

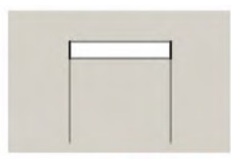

LALBA
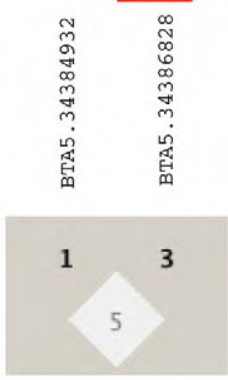

(C)
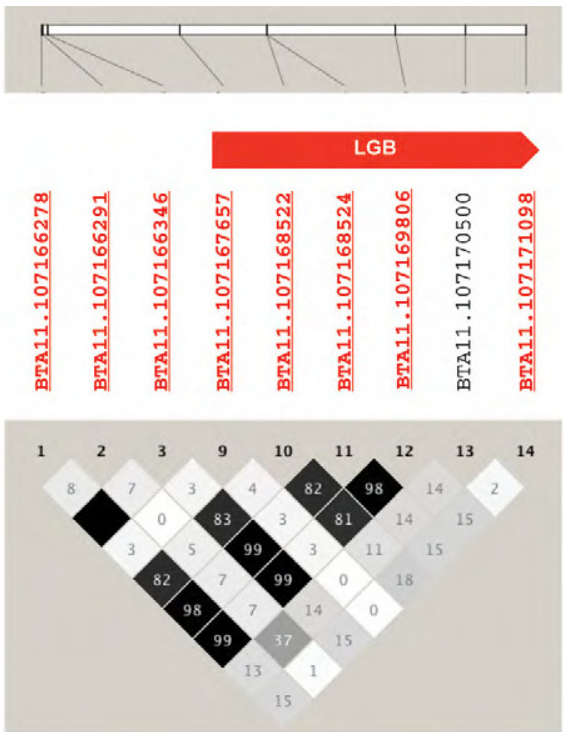

(B)
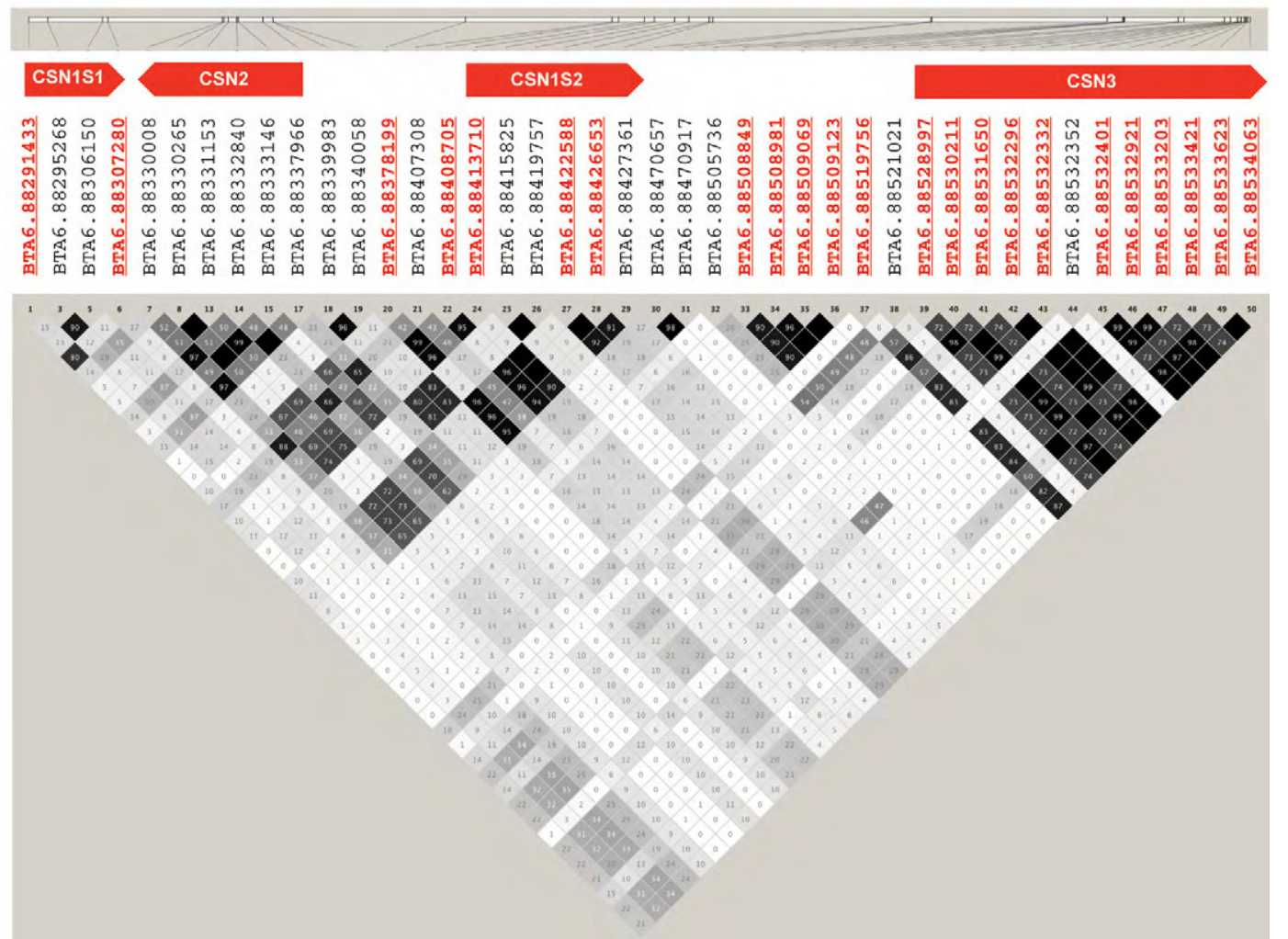

Figure 1. Linkage disequilibrium (LD) between genotyped SNP. In each panel, the top horizontal rectangle shows the relative chromosomal locations of each SNP on a bovine chromosome. Each vertical bar represents an SNP. Genes are shown by red horizontal bars with the direction of the arrowhead indicating the transcriptional strand. The bottom box shows a heatmap of linkage disequilibrium $\left(\mathrm{r}^{2}\right)$, with SNP IDs shown on top of the heatmap. Darker shading indicates stronger LD. The SNP are shown on 3 separate chromosomes, including the casein cluster on BTA6 (B), LALBA (A), and $L G B(\mathrm{C})$. The ID of SNP that showed significant associations with any trait are boldface and underlined (colored in red). Color version available in the online PDF. 
Table 3. Additive genetic variance of milk composition traits

\begin{tabular}{|c|c|c|c|}
\hline Trait & $\begin{array}{c}\text { Total } \\
\operatorname{variance}^{1}\left(\sigma_{\text {total }}^{2}\right)\end{array}$ & $\begin{array}{l}\text { Additive genetic } \\
\text { variance }\left(\sigma_{a}^{2}\right)\end{array}$ & $\begin{array}{c}\text { Proportion } \\
\text { of } \sigma_{a}^{2} \text { in } \sigma_{\text {total }}^{2}(\%)\end{array}$ \\
\hline$\alpha_{\mathrm{S}^{-}} \mathrm{CN}(\mathrm{mg} / 100 \mu \mathrm{L})$ & $1.03 \times 10^{-2}$ & $3.43 \times 10^{-3}$ & 33.2 \\
\hline$\beta-\mathrm{CN}(\mathrm{mg} / 100 \mu \mathrm{L})$ & $2.27 \times 10^{-2}$ & $7.44 \times 10^{-3}$ & 32.8 \\
\hline$\kappa-\mathrm{CN}(\mathrm{mg} / 100 \mu \mathrm{L})$ & $5.39 \times 10^{-3}$ & $3.57 \times 10^{-3}$ & 66.3 \\
\hline$\alpha-\mathrm{LA}(\mathrm{mg} / 100 \mu \mathrm{L})$ & $2.42 \times 10^{-4}$ & $7.90 \times 10^{-5}$ & 32.6 \\
\hline$\beta-\mathrm{LG}(\mathrm{mg} / 100 \mu \mathrm{L})$ & $1.47 \times 10^{-2}$ & $1.01 \times 10^{-2}$ & 69.1 \\
\hline Total protein $(\mathrm{mg} / 100 \mu \mathrm{L})$ & $1.25 \times 10^{-1}$ & $4.95 \times 10^{-2}$ & 39.6 \\
\hline$\alpha_{S^{-}} \mathrm{CN}$ to $\beta-\mathrm{CN}$ ratio & $1.94 \times 10^{-3}$ & $1.29 \times 10^{-3}$ & 66.7 \\
\hline
\end{tabular}

were all purebred Holsteins, who predominantly carried the A allele. On the other hand, Jerseys carried the G allele at a much higher frequency. Cows heterozygous for this SNP produced a significantly higher concentration of $\alpha_{S^{-}} \mathrm{CN}$ in their milk than homozygous A animals (Supplemental Table 1, http://www.journalofdairy science.org/).

Five SNP (BTA6.88378199, BTA6.88408705, BTA6.88413710, BTA6.88422588, and BTA6.88426653) in almost complete LD (all pair-wise $\mathrm{r}^{2}>0.9$, Figure 1) were found to be significantly associated with $\beta-\mathrm{CN}$ concentration (Supplemental Table 1, http://www. journalofdairyscience.org). Except BTA6.88378199 and BTA6.88408705, which are about 31 and $317 \mathrm{bp}$ upstream of the CSN1S2 gene, respectively, the other $3 \mathrm{SNP}$ are located within introns of the CSN1S2 gene.
Interestingly, BTA6.88378199 also appeared to be in moderate LD $\left(\mathrm{r}^{2}>0.6\right)$ with several SNP within the CSN2 gene, the product of which $(\beta-\mathrm{CN})$ this SNP was associated with (Figure 1).

Sixteen SNP were significantly associated with $\kappa-\mathrm{CN}$ concentration (Figure 2, Supplemental Table 1, http:// www.journalofdairyscience.org). However, these SNP could be partitioned into 2 clusters with extremely high LD (Figure 1). One was located approximately $12.5 \mathrm{~kb}$ upstream of the CSN3 gene, and included BTA6.88508849, BTA6.88508981, BTA6.88509069, and BTA6.88509123. The other cluster included 12 SNP within the CSN3 gene, among which 2 were amino acid changing SNP (BTA6.88532296 and BTA6.88532332) that differentiate the $\mathrm{A}$ and $\mathrm{B}$ variants of $\kappa-\mathrm{CN}$ (Table $2)$.

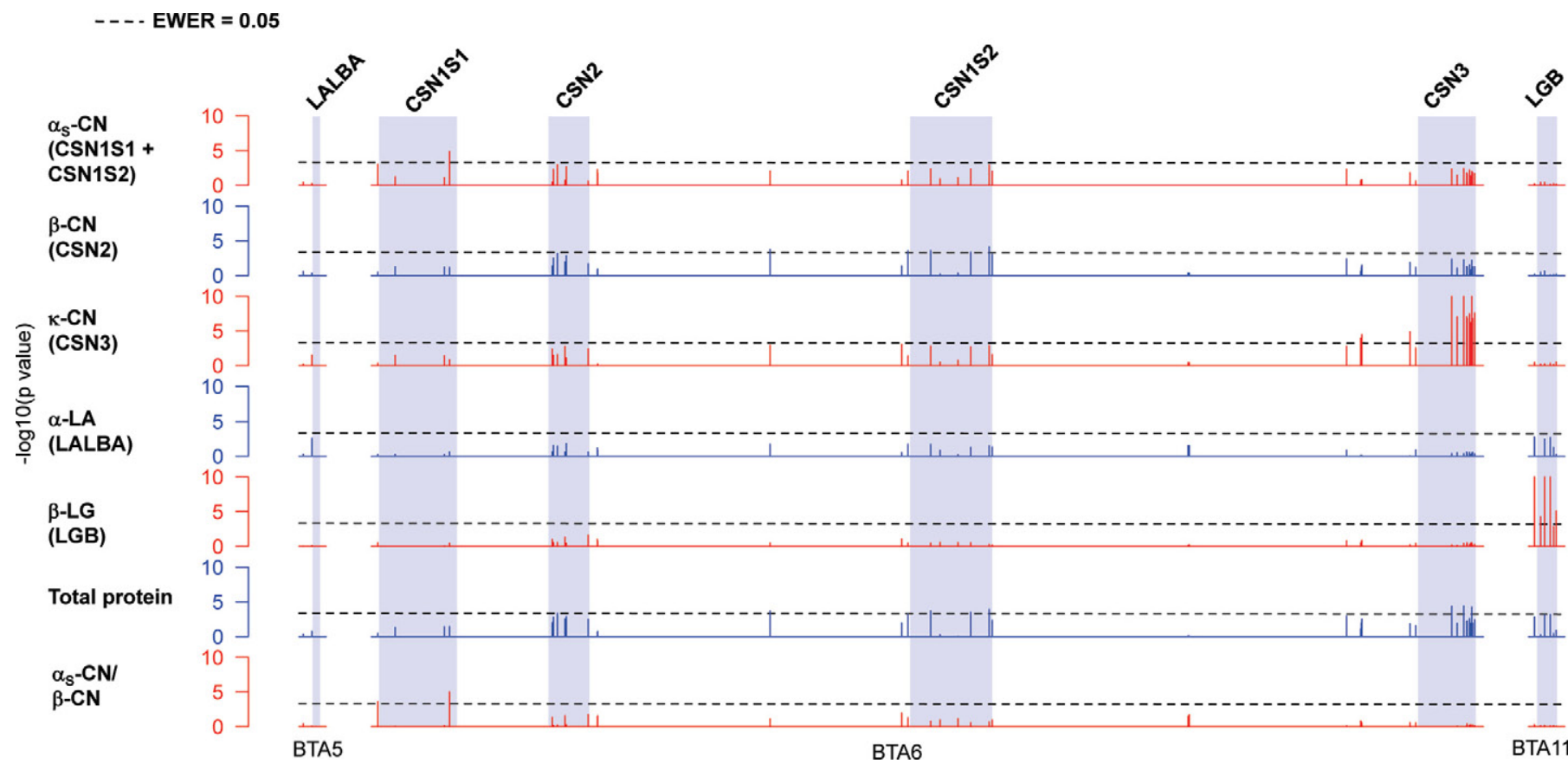

Figure 2. Association between milk protein gene variants and individual protein concentrations. Each vertical bar represents an SNP tested for association with its height being the $-\log _{10}(P$-value $)$ of the type III $F$-test and x-axis position being the chromosomal location; SNP with $P$-values $<10^{-10}$ are plotted as $10^{-10}$. Light blue (shaded) rectangles represent the boundaries of milk protein genes. Horizontal dashed lines are the experiment-wise error rate $=0.05$ thresholds. Color version available in the online PDF. 
Table 4. Variance explained by SNP selected by a stepwise selection procedure for milk protein composition traits

\begin{tabular}{|c|c|c|c|c|c|c|c|}
\hline Trait & $\begin{array}{l}\text { Significant } \\
\text { SNP }\end{array}$ & $\begin{array}{l}\text { Type III } \\
P \text {-value }\end{array}$ & $\begin{array}{l}\text { Genotype } \\
\text { (no.) }\end{array}$ & $\begin{array}{l}\text { Estimated } \\
\text { effect (SE) }\end{array}$ & $\sigma_{S N P}^{2}$ & $\sigma_{\text {total }}^{2}$ & $\begin{array}{c}\sigma_{S N P}^{2} / \sigma_{\text {total }}^{2} \\
(\%)\end{array}$ \\
\hline$\alpha_{S^{-}} \mathrm{CN}(\mathrm{mg} / 100 \mu \mathrm{L})$ & BTA6.88307280 & $<0.0001$ & $\begin{array}{l}\text { AG }(78) \\
\text { AA }(184)\end{array}$ & $\begin{array}{l}0.0578(0.0130) \\
0\end{array}$ & 0.0016 & 0.0114 & 14.0 \\
\hline$\beta-\mathrm{CN}(\mathrm{mg} / 100 \mu \mathrm{L})$ & BTA6.88426653 & $<0.0001$ & $\begin{array}{l}\text { GG (31) } \\
\text { AG (114) } \\
\text { AA (113) }\end{array}$ & $\begin{array}{l}0.0650(0.0300) \\
0.0817(0.0184) \\
0\end{array}$ & 0.0018 & 0.0229 & 7.9 \\
\hline$\kappa-\mathrm{CN}(\mathrm{mg} / 100 \mu \mathrm{L})$ & BTA6.88528997 & $<0.0001$ & $\begin{array}{l}\text { AA }(50) \\
\text { AC }(150) \\
\text { CC }(68)\end{array}$ & $\begin{array}{l}0.2655(0.0287) \\
0.1013(0.0119) \\
0\end{array}$ & 0.0025 & 0.0066 & 37.9 \\
\hline$\beta-\mathrm{LG}(\mathrm{mg} / 100 \mu \mathrm{L})$ & BTA11.107169806 & $<0.0001$ & $\begin{array}{l}\text { CC }(44) \\
\text { CT }(129) \\
\text { TT }(92)\end{array}$ & $\begin{array}{l}-0.2379(0.0146) \\
-0.1106(0.0010) \\
0\end{array}$ & 0.0140 & 0.0215 & 65.1 \\
\hline \multirow[t]{2}{*}{ Total protein $(\mathrm{mg} / 100 \mu \mathrm{L})$} & BTA6.88378199 & 0.0007 & $\begin{array}{l}\text { CC (27) } \\
\text { CT (107) } \\
\text { TT (113) }\end{array}$ & $\begin{array}{l}0.1677(0.0665) \\
0.1465(0.0402) \\
0\end{array}$ & 0.0074 & 0.1326 & 5.6 \\
\hline & BTA6.88531650 & 0.0003 & $\begin{array}{l}\text { GG }(43) \\
\text { AG }(143) \\
\text { AA }(61)\end{array}$ & $\begin{array}{l}0.2551(0.0621) \\
0.1361(0.0467) \\
0\end{array}$ & 0.0143 & 0.1326 & 10.8 \\
\hline$\alpha_{\mathrm{S}}-\mathrm{CN}$ to $\beta$-CN ratio & BTA6.88307280 & $<0.0001$ & $\begin{array}{l}\text { AG }(78) \\
\text { AA }(184)\end{array}$ & $\begin{array}{l}0.0240(0.0053) \\
0\end{array}$ & 0.0002 & 0.0020 & 10.0 \\
\hline
\end{tabular}

Although no SNP were significantly associated with $\alpha$-LA, 8 were found to be associated with $\beta$-LG concentration (Supplemental Table 1; http://www. journalofdairyscience.org/). Among the latter were the BTA11.107168522 SNP, a synonymous mutation, and the BTA11.107168524 and BTA11.107169806 SNP, nonsynonymous mutations that differentiated the A and $B$ variants of $\beta$-LG (Table 2).

Seven SNP dispersed along the casein cluster on BTA6 were found to be associated with total protein concentration (Figure 2; Supplemental Table 1, http:// www.journalofdairyscience.org). Interestingly, all of these SNP were also significantly associated with at least one individual protein concentration (Figure 2). The most significant association for total protein was with SNP BTA6.88531650 located within the CSN3 gene.

Two SNP (BTA6.88291433 and BTA6.88307280) located within the CSN1S1 gene were associated with $\alpha_{\mathrm{S}^{-}}$ to $\beta$-CN ratio (Supplemental Table 1; available online at http://www.journalofdairyscience.org/). Although BTA6.88307280 was also highly associated with $\alpha_{S^{-}} \mathrm{CN}$ concentration, BTA6.88291433 $(P=0.0009)$ appeared to be associated with $\alpha_{\mathrm{S}}$-CN but did not reach the significance threshold after multiple testing correction. Interestingly, SNP that were associated with $\beta-\mathrm{CN}$ concentration did not appear to affect the $\alpha_{S^{-}}$to $\beta-\mathrm{CN}$ ratio.

\section{Variation Explained by Significant SNP for Protein Composition Traits}

Because multiple linked and independent SNP were found to be associated with each trait, a stepwise selec- tion procedure was used to find the best subset of SNP that explained the variation in each trait. The stepwise selection procedure selected a single significant SNP for $\alpha_{\mathrm{S}}-\mathrm{CN}, \beta-\mathrm{CN}, \kappa-\mathrm{CN}, \beta-\mathrm{LG}$, and $\alpha_{\mathrm{S}^{-}} \mathrm{CN}$ to $\beta-\mathrm{CN}$ ratio, and 2 SNP for total protein (Table 4), which may be explained by the fact that substantial correlation was found between SNP genotypes among significant SNP; therefore, accounting for one SNP eliminated the effect of the other SNP. An example is the significant SNP in the $C S N 3$ gene that appeared to be in very high $\mathrm{LD}$ (Figure 1).

Remarkably, single significant SNP explained 14.0, $7.9,37.9,65.1$, and $10.0 \%$ of the phenotypic variation for $\alpha_{S^{-}} \mathrm{CN}, \beta-\mathrm{CN}, \kappa-\mathrm{CN}, \beta-\mathrm{LG}$, and $\alpha_{S^{-}} \mathrm{CN}$ to $\beta-\mathrm{CN}$ ratio, respectively (Table 4). Moreover, 2 SNP together explained $16.4 \%$ of the phenotypic variation of total protein (Table 4). In particular, the BTA11.107169806 SNP in the $L G B$ gene explained the majority of the variation for $\beta$-LG. These results clearly suggest the existence of major QTL for milk protein composition traits.

\section{DISCUSSION}

This study investigated the association between DNA variants in milk protein genes and individual milk protein concentrations in a resource population consisting of purebred Holstein and (Holstein $\times$ Jersey) $\times$ Holstein crossbred animals. Several SNP were significantly associated with 6 milk protein composition traits. Importantly, the significant SNP explained a large proportion of the phenotypic variation of milk composition.

Several previous studies have reported associations between milk protein gene variants and protein com- 
position, including traits such as casein-to-total-protein ratio (Lundén et al., 1997) or protein percentage (Bovenhuis et al., 1992), and more recently, relative concentrations of individual proteins (Heck et al., 2009). However, most previous studies looked at a limited number of variants, typically those with amino acidchanging alleles. In contrast, the current study tested a comprehensive list of $53 \mathrm{DNA}$ variants within and close to the milk protein genes. In addition, this association study was performed in a unique resource population that consisted of both purebred Holstein and (Holstein $\times$ Jersey $) \times$ Holstein backcross cows. The introduction of alleles from the Jersey breed, especially those with breed-specific prevalence, was expected to induce genetic variation thus facilitating the mapping effort.

A substantial additive genetic component was found for all 7 protein composition traits studied (Table 3). It should be noted that this estimation was based on an assumption that the covariance between 2 animals with the same degree of relatedness was the same regardless of their breed identity in a multibreed population. However, this assumption may be violated (Lo et al., 1993). To test this possibility, the data were analyzed separately for Holsteins and crossbreds. This, however, significantly reduced the sample size for each subset of the data. Nevertheless, both sub-populations showed remarkably substantial proportions of additive genetic components, as was seen when they were combined. Similar results have been reported previously, although individual protein components were expressed as ratios to total protein (Schopen et al., 2009).

Despite the fact that milk protein composition traits are highly heritable, their genetic architectures remain unclear. In particular, interactions between genes and interactions between genes and environments may be important for the phenotypic variation but have never been studied for milk protein composition traits. A recent genome-wide association study in the Dutch Holstein population provided a first hint at the distribution of QTL effects and locations (Schopen et al., 2011). Although multiple chromosomal regions were typically found to be associated with each milk protein, the strongest associations uniformly co-localized with chromosomal regions where the proteins' structural genes themselves were located (Schopen et al., 2011). The fact that SNP in genes were associated with proteins that were expressed from the same gene suggested that the QTL were likely to have cis effects. However, particularly for the 4 casein genes that are closely linked (Figure 1), it was unclear whether or not the cis effect was confined to the same gene.

The fact that few QTL were detected in a relatively large sample (Schopen et al., 2011) suggested that only a limited number of QTL exist for these traits or that many more QTL with very small effects remain to be identified. The former appeared to be a more plausible explanation because significant SNP in the genomewide association study explained large proportions of phenotypic variance (Schopen et al., 2011). Results of this study also suggested the existence of major QTL for milk protein composition traits. Single SNP explained a substantial fraction of phenotypic variation for $\alpha_{\mathrm{S}}-\mathrm{CN}, \beta-\mathrm{CN}, \kappa-\mathrm{CN}, \beta-\mathrm{LG}$, and $\alpha_{\mathrm{S}}-\mathrm{CN}$ to $\beta-\mathrm{CN}$ ratio, as did 2 SNP for total protein (Table 3). However, the possibility of multiple linked QTL being tagged by single SNP cannot be excluded. In particular, SNP in the casein cluster on BTA6 appeared to be in considerable LD (Figure 1), and haplotypes extend to a very long range under artificial selection (Qanbari et al., 2010). For this reason, causal mutations remain to be identified because many SNP in high LD would explain the phenotypic variation equally well. The relatively large extent of LD and small genetic diversity in cattle may not permit identification of causal variants or separation of multiple linked QTL unless an extremely large sample can be collected to enrich the number of recombination events.

A unique feature of the resource population used in this study was the introduction of Jersey chromosomes by crossbreeding. Because a large difference in milk components exists between Holstein and Jersey (Schutz et al., 1990), the introduction of Jersey alleles is expected to increase genetic diversity and facilitate gene mapping. One example was the significant association between the BTA6.88307280 SNP in CSN1S1 with $\alpha_{S^{-}} \mathrm{CN}$, and $\alpha_{S^{-}} \mathrm{CN}$ to $\beta-\mathrm{CN}$ ratio. The difference in allele frequencies of the $\mathrm{G}$ allele in purebred Holstein and (Holstein $\times$ Jersey $) \times$ Holstein backcross suggested a breed differentiation at this SNP location. Indeed, the $\mathrm{G}$ allele identifies the $\mathrm{C}$ variant of $\alpha_{\mathrm{S} 1}-\mathrm{CN}$, which has been previously shown to be very rare in the Holstein population (Formaggioni et al., 1999; Heck et al., 2009). It is likely that the association between this SNP and $\alpha_{S^{-}} \mathrm{CN}$ would have not been detected in a purebred Holstein population.

Using phenotypes expressed as the proportions of individual proteins among total protein, previous studies often found associations between milk protein variants and multiple protein component traits (Heck et al., 2009). This was expected because a change in the proportion of one milk protein would inevitably affect the proportion of another milk protein. Associations between milk protein variants in one gene and the protein expression of another gene have not been observed in this study, partly because absolute, rather than relative, protein concentrations were phenotyped. Nevertheless, SNP that were associated with $\beta$-CN were located within the CSN1S2 gene and intergenic 
region (Figure 1). However, this does not necessarily imply that these SNP would change the concentration of $\beta$-CN due to considerable linkage disequilibrium between the CSN1S2 and CSN2 genes. Indeed, haplotypes encompassing the CSN2 and CSN3 gene have been shown to affect milk protein composition, suggesting that units of inheritance may span a large genomic region (Bonfatti et al., 2010).

Several of the significant SNP identified in this study have been previously shown to affect milk protein components. The C allele of the BTA6.88532296 SNP and the A allele of the BTA6.88532332 SNP encode threonine at the 136th and aspartic acid at the 148th AA of $\kappa$-CN, respectively (Table 2), which together identify the $\kappa-\mathrm{CN}$ A variant. These 2 alleles were associated with lower $\kappa-\mathrm{CN}$ concentration in our study as well as with lower $\kappa$-CN proportion in a previous study (Heck et al., 2009). Meanwhile, the B variant of the $L G B$ gene has been consistently shown to be associated with lower $\beta$-LG proportion in milk protein (Bobe et al., 1999). The same association was also observed in the current study, where the completely linked G allele of BTA11.107168524 and the C allele of BTA11.107169806 were associated with decreased $\beta$-LG concentration (Table 2, Supplemental Table 1, http://www.journalofdairyscience.org). Indeed, most previous studies investigating association between milk protein gene variants and milk protein composition were limited to protein-coding variants. In addition to significant SNP that changed amino acids and SNP that were in high LD with them, we also identified SNP that were not in protein-coding regions. For example, BTA6.88378199 that was found to be associated with $\beta$-CN was an intergenic SNP located between the CSN2 and CSN1S2 genes.

The exact mechanisms by which polymorphisms change protein expression remain to be revealed. For example, at least 2 possibilities explain how nonsynonymous SNP can cause changes in protein expression. On one hand, they can be linked with regulatory variants that may alter gene expression. In this case, the association reflects genotypic correlation rather than causality. On the other hand, changes in AA sequence may affect translation efficiency and protein stability. A combination of mRNA and protein expression analyses may help to distinguish different possibilities.

The identification of large-effect SNP markers for individual protein concentrations has important applications. Current selection on milk composition is limited to overall protein and fat. Phenotyping animals for individual protein concentrations can be economically prohibitive, which limits its large-scale application. However, many desirable properties of milk are related to individual protein concentrations or ratios between them. For example, in this study, we identified SNP associated with the ratio of $\alpha_{S}-\mathrm{CN}$ to $\beta$-CN. It has been shown that a lower $\alpha_{S}-\mathrm{CN}$ to $\beta-\mathrm{CN}$ ratio is associated with poorer coagulation in cheese making (St-Gelais and Hache, 2005). Single nucleotide polymorphism markers that explain large proportions of phenotypic variation for milk protein composition traits may be very useful in selecting animals that produce milk with desired processing and manufacturing properties.

In summary, a comprehensive association study between milk protein gene variants and milk protein composition traits was performed in a unique resource population consisting of purebred Holstein and (Holstein $\times$ Jersey $) \times$ Holstein crossbred cows. Single nucleotide polymorphisms were found to be significantly associated with 6 of the protein composition traits $\left(\alpha_{S^{-}} \mathrm{CN}\right.$, $\beta-\mathrm{CN}, \kappa-\mathrm{CN}, \beta-\mathrm{LG}$, total protein, and $\alpha_{S^{-}} \mathrm{CN}$ to $\beta-\mathrm{CN}$ ratio). The results indicate substantial heritable proportions of the phenotypic variation as well as major effects caused by a few SNP.

\section{ACKNOWLEDGMENTS}

This study was supported by USDA Hatch grant No. WIS-142-PRJ16JH from the University of WisconsinMadison.

\section{REFERENCES}

Auldist, M. J., K. A. Johnston, N. J. White, W. P. Fitzsimons, and M. J. Boland. 2004. A comparison of the composition, coagulation characteristics and cheesemaking capacity of milk from Friesian and Jersey dairy cows. J. Dairy Res. 71:51-57.

Auldist, M. J., B. J. Walsh, and N. A. Thomson. 1998. Seasonal and lactational influences ion bovine milk composition in New Zealand. J. Dairy Res. 65:401-411.

Bailey, K. W., C. M. Jones, and A. J. Heinrichs. 2005. Economic returns to Holstein and Jersey herds under multiple component pricing. J. Dairy Sci. 88:2269-2280.

Barrett, J. C., B. Fry, J. Maller, and M. J. Daly. 2005. Haploview: Analysis and visualization of LD and haplotype maps. Bioinformatics 21:263-265.

Bobe, G., D. C. Beitz, A. E. Freeman, and G. L. Lindberg. 1999. Effect of milk protein genotypes on milk protein composition and its genetic parameter estimates. J. Dairy Sci. 82:2797-2804.

Bonfatti, V., G. Di Martino, A. Cecchinato, D. Vicario, and P. Carnier. 2010. Effects of $\beta$ - $\kappa$-casein (CSN2-CSN3) haplotypes and $\beta$-lactoglobulin (BLG) genotypes on milk production traits and detailed protein composition of individual milk of Simmental cows. J. Dairy Sci. 93:3797-3808.

Bovenhuis, H., J. A. M. Van Arendonk, and S. Korver. 1992. Associations between milk protein polymorphisms and milk production traits. J. Dairy Sci. 75:2549-2559.

Caroli, A. M., S. Chessa, and G. J. Erhardt. 2009. Invited review: Milk protein polymorphisms in cattle: Effect on animal breeding and human nutrition. J. Dairy Sci. 92:5335-5352.

Formaggioni, P., A. Summer, M. Malacarne, and P. Mariani. 1999. Milk protein polymorphism: Detection and diffusion of the genetic variants in Bos genus. Pages 127-165 in Ann. Fac. Med. Vet. Univ. Parma. XIX, Parma, Italy.

Gao, X., J. Starmer, and E. R. Martin. 2008. A multiple testing correction method for genetic association studies using correlated single nucleotide polymorphisms. Genet. Epidemiol. 32:361-369. 
Ganai, N. A., H. Bovenhuis, J. A. M. van Arendonk, and M. H. Visker. 2009. Novel polymorphisms in the bovine beta-lactoglobulin gene and their effects on beta-lactoglobulin protein concentration in milk. Anim. Genet. 40:127-133.

Heck, J. M. L., A. Schennink, H. J. F. van Valenberg, H. Bovenhuis, M. H. P. W. Visker, J. A. M. van Arendonk, and A. C. M. van Hooijdonk. 2009. Effects of milk protein variants on the protein composition of bovine milk. J. Dairy Sci. 92:1192-1202.

Kaminski, S., A. Ahman, A. Rusc, E. Wojcik, and T. Malewski. 2005. MilkProtChip-A microarray of SNPs in candidate genes associated with milk protein biosynthesis: Development and validation. J. Appl. Genet. 46:45-58.

Lo, L. L., R. L. Fernando, and M. Grossman. 1993. Covariance between relatives in multibreed populations: Additive model. Theor. Appl. Genet. 87:423-430.

Lucey, J. A., and J. Kelly. 1994. Cheese yield. J. Soc. Dairy Technol. $47: 1-14$.

Lundén, A., M. Nilsson, and L. Janson. 1997. Marked effect of $\beta$-lactoglobulin polymorphism on the ratio of casein to total protein in milk. J. Dairy Sci. 80:2996-3005.

Maltecca, C., K. A. Weigel, H. Khatib, M. Cowan, and A. Bagnato. 2009. Whole-genome scan for quantitative trait loci associated with birth weight, gestation length and passive immune transfer in a Holstein $\times$ Jersey crossbred population. Anim. Genet. 40:27-34.

Ng-Kwai-Hang, K., J. F. Hayes, J. E. Moxley, and H. G. Monardes. 1987. Variation in milk protein concentrations associated with genetic polymorphism and environmental factors. J. Dairy Sci. 70:563-570

Nilsen, H., H. G. Olsen, B. Hayes, E. Sehested, M. Svendsen, T. Nome, T. Meuwissen, and S. Lien. 2009. Casein haplotypes and their association with milk production traits in Norwegian Red cattle. Genet. Sel. Evol. 41:24.
Qanbari, S., E. C. Pimentel, J. Tetens, G. Thaller, P. Lichtner, A. R. Sharifi, and H. Simianer. 2010. A genome-wide scan for signatures of recent selection in Holstein cattle. Anim. Genet. 41:377-389.

R Development Core Team. 2010. R: A Language and Environment for Statistical Computing. R Foundation for Statistical Computing, Vienna, Austria.

SAS Institute. 2009. SAS 9.1.3 Help and Documentation. SAS Institute Inc., Cary, NC.

Schopen, G. C., M. H. Visker, P. D. Koks, E. Mullaart, J. A. van Arendonk, and H. Bovenhuis. 2011. Whole-genome association study for milk protein composition in dairy cattle. J. Dairy Sci. 94:3148-3158.

Schopen, G. C. B., J. M. L. Heck, H. Bovenhuis, M. H. P. W. Visker, H. J. F. van Valenberg, and J. A. M. van Arendonk. 2009. Genetic parameters for major milk proteins in Dutch Holstein-Friesians. J. Dairy Sci. 92:1182-1191.

Schutz, M. M., L. B. Hansen, G. R. Steuernagel, and A. L. Kuck. 1990. Variation of milk, fat, protein, and somatic cells for dairy cattle. J. Dairy Sci. 73:484-493.

St-Gelais, D., and S. Hache. 2005. Effect of beta-casein concentration in cheese milk on rennet coagulation properties, cheese composition and cheese ripening. Food Res. Int. 38:523-531.

Tauzin, J., L. Miclo, and J. L. Gaillard. 2002. Angiotensin-I-converting enzyme inhibitory peptides from tryptic hydrolysate of bovine $\alpha_{S 2^{-}}$ casein. FEBS Lett. 531:369-374.

Wedholm, A., E. Hallen, L. B. Larsen, H. Lindmark-Mansson, A. H. Karlsson, and T. Allmere. 2006. Comparison of milk protein composition in a Swedish and a Danish dairy herd using reversed phase HPLC. Acta Agric. Scand. A Anim. Sci. 56:8-15. 